\title{
Omniscience and Semantic Information
}

\author{
Bernardo Alonso \\ Universidade Federal do Mato Grosso \\ Department of Philosophy \\ Cuiaba, $M A$ \\ Brazil \\ berr.alonso@gmail.com
}

Article info

CDD: 120

Received: 01.07.2017; Accepted: 16.10.2017

DOI: http://dx.doi.org/10.1590/0100-6045.2017.V40N4.BA

\section{Keywords: \\ Logical Omniscience Problem \\ Knowledge \\ Semantic Informatio}

\begin{abstract}
First, I consider a few motivations to idealize epistemic logics ${ }^{1}$ in such a degree that brings up the problem of logical omniscience [LOP]. I argue that the main motivation to hold omniscience is of a philosophical-scientific ${ }^{2}$ background (Stalnaker 1991), in the sense philosophers have a not so peculiar way of investigating underlying mechanisms, i.e., the interaction of several different components of complex systems may be better understood in isolation, even if such components are not found isolated in a realistic context. It is defended that the implicit and explicit knowledge distinction (Fagin and Halpern 1988) is compatible that view since idealizations made by modal epistemic logic are so strong that the agents they describe hardly have anything in common with real agents. I conclude by showing how LOP can be accommodated in the logic of being informed (Floridi 2006) using the Inverse Relationship Principle (Barwise and Seligman 1997).
\end{abstract}

${ }^{1}$ Epistemic modal logics and the logic of being informed may collapse in many scenarios, as shown in previous articles (blind review omitted).

${ }^{2}$ Does not necessarily imply in a naturalized epistemology a la (Quine 1981). For a plea for non-naturalism as constructionism see (Floridi 2017).

Manuscrito - Rev. Int. Fil. Campinas, v. 40, n. 4, pp. 77-96, out.-dez. 2017. 


\section{Introduction}

In general, the problem of logical omniscience is connected to the concept that if an agent knows all formulas in a set $\Gamma$ and $\varphi$ follows logically from $\wp$, then the agent also knows that $\varphi$ (Fagin et al 1995). Thus considered, an agent knows all theorems and all logical consequences of a sentence that he knows (taking $\wp$ to consist of a single sentence). Such notion may come in different flavours:

- Knowledge of valid formulas: the agent knows all logical truths (necessitation, NEC rule);

- Closure under logical implication: if the agent $a$ knows $\varphi$ and if $\varphi$ logically implies $\psi$ (i.e. $\varphi \rightarrow \psi$ is valid), then the agent a knows $\psi$ (monadic, MON rule);

- Closure under logical equivalence: if the agent $a$ knows that $\varphi$ and if $\varphi$ and $\psi$ are logically equivalent (i.e. $\varphi \leftrightarrow \psi$ is valid), then the agent $a$ knows $\psi$ (congruence, CGR rule);

- Closure under material implication: if the agent $a$ knows that $\varphi$ and if the agent $a$ knows that $(\varphi \rightarrow \psi)$, then the agent $a$ knows that $\psi(\mathrm{K}$ axiom);

- Closure under conjunction: if the agent $a$ knows that $\varphi$ and if the agent $a$ knows that $\psi$, then the agent $a$ knows that $\varphi \wedge \psi(\mathrm{C}$ rule).

One of the main purposes of language is to transmit information about the world. Where $\mathrm{p}$ is any sentence used for that purpose, it seems natural to think of content as the information that is semantically encoded in it. With this account of information at hand, we can think of the content of a sentence as a set of possible worlds. The problem of logical omniscience in standard modal epistemic and doxastic logics is linked to the axiom $\mathrm{K}$ (distributive property and deductive cogency). Roughly, it says that if an agent knows that $\varphi$, and if an agent knows that $\varphi$ implies $\psi$, then knowing that $\varphi$ implies knowing that $\psi$.

Manuscrito - Rev. Int. Fil. Campinas, v. 40, n. 4, pp. 77-96, out.de\%. 2017. 
However, it seems a bit unrealistic to attribute to agents a sizeable deductive power to the point of knowing (or believing) all the logical consequences of what they know (or believe)3. As Hintikka (1962) taught us, it is an idealization that serves logic and it is precisely from the tension between the assumptions of an ideal theory and the intended application within a certain logic that this problem arises - the problem of logical omniscience. Hintikka summarizes his approach by holding that "in order to speak of what a certain person a knows and does not know, we have to assume a class ('space') of possibilities" (Hintikka 2003:19). There seem to be two ways of trying to reconcile the fact that people do not believe in all the logical consequences of their beliefs with a theory that says they do believe. The first is to restrict the range of its application to imaginary believers, an idealized special type with virtually unlimited resources of memory, computational capacity and speed (Lemmon 1959). The other way would be to consider the domain of the application of such logic in an unrestricted way, which includes not only idealized agents. In this second type of conciliation attempt we would interpret the concepts of "belief" or "knowledge" modelled by the theory in a special sense, where the difference between the ideal and the real is explained by the difference between "knowledge" in an ordinary sense and "knowledge" in a technical sense.

On one hand we would have an implicit knowledge (Levesque 1984), which would be that of the technical meaning, which by definition includes all deductive consequences irrespective of whether or not the agent becomes aware of such consequences. On the other hand the ordinary sense tells us that we do not really know all the logical consequences of what we know. Note that implicit knowledge (or belief) differs from a "possible" knowledge, for the latter may have no relation to the current (explicit) belief of a subject. It is important to notice that implicit knowledge or beliefs we possess only in principle and even the most ignorant among us is deductively omniscient in principle (Van Benthem and Velázquez-Quesada 2010). The interesting question here is what should count as cases of knowledge.

\footnotetext{
${ }^{3}$ Notwithstanding important differences between the notions of belief and knowledge, especially in relation to truth conditions - a true belief in the present world is not necessarily preserved if given circumstances are modified, while it is assumed that knowledge (and information) in the present world is stable between circumstances radically different (Hendricks 2006) - in general the problem of logical omniscience affects both notions in a similar way.
} 


\section{Motivations}

Is there any positive reason for idealization in epistemic logic? According to Stalnaker (1991) one of the main motivations is the investigation of underlying mechanisms, i.e., the interaction of several different components of complex systems may be better understood in isolation, even if such components are not found isolated in a realistic context. In scientific practice we are used to this: to isolate certain components from factors that would undesirably affect the behaviour of certain experiments, ranging from useful "under standard conditions for temperature and pressure", to historical Galileo's "frictionless plane", Atwood's machine and its "massless, inextensible string and an ideal massless pulley", "perfect vacuum", the notion of "isolated systems", and even anecdotal oversimplified "spherical cows" or "assume a can opener" from economics theories. When we construct ideal models we want to understand, among other things, the reason for certain limitations that we find in real situations. Another example that is closer to the subject matter in question is Chomsky's (1965) "performance-competence" distinction, designed to isolate a psychological capacity that is language-specific. In his framework "performance" is what the speakers actually express, it is a surface phenomenon, and "competence" is the ideal language system that enables speakers to produce and understand an infinite number of sentences in their language, and to distinguish grammatical sentences from ungrammatical sentences. This is unaffected by "grammatically irrelevant" conditions such as speech errors. In Chomsky's view, competence can be studied independently of language use. It does not matter if one agree with his theory, the point being taken here is that this sort of idealization is often used in intellectual enterprises of various grounds.

Baggini and Fosl (2010) points out that there is a considerably small but effective set of theoretical tools that philosophers find at their disposal. Among the tools we have to help in the formulation and construction within the various stages of philosophical practice, let us highlight two types which, despite their subtle differences, generally work together and are often not distinguished in contemporary philosophy of analytical influence. They are: the work of intuitions and the thought experiments. When ideas, concepts or notions which we encounter in philosophy and science are too difficult to convey and to be understood - either by its structural complexity or level of abstraction - both philosophers and scientists appeal to metaphors and images. The term intuition pump was coined by Daniel Dennett in a critique of John 
Searle's Chinese Room Argument (Dennet 1998). According to Dennett, despite the name "Chinese Room Argument" (Searle 1980), there was no argument in Searle's text against what in fact was involved in the notion of artificial intelligence, namely against the semantic understanding of the combination of symbols by a machine. For Dennett the picture presented was nothing more than a mere product of the so called intuition, he thinks that Searle's thought experiment is what he calls a "boom crutch" - a faulty intuition pump. Although the term being initially coined somewhat disparagingly, everyone who writes scientific or philosophical texts makes use of intuition pumps, including Dennett, of course. These intuition boosts, if well used, can greatly contribute to the understanding of a problem, and it is not by chance that the Chinese room argument has yielded more than thirty years of productive academic debates. When we hear all that talk about inputs and outputs from philosophy of mind, psychology and cognitive science functionalist theories, our attention and visualization of what the theory really wants to show can be somewhat blurred. But when we think of the brain as a part of a computer's hardware and think of the mind as a kind of software, a program that runs on hardware, it becomes easier to grasp what is at stake.

Let's now turn to thought experiments. There is a long-standing dispute between philosophers who think there is an important continuity between philosophy and science and those who think that philosophy is a totally different form of inquiry and way of seeing the world. Such division, thus placed, makes it seem that there are two rather distinct groups of people who produce philosophy. On one hand we would have boring, squared philosophers with scientific aspirations and lacking creativity. On the other side we would have creative philosophers with artistic aspirations and who make use of a poetic and elaborate language. It is curious to notice that today the philosophers of the so-called "analytic" tradition, regarded as brutalized by the coldness of logic, are those who are admittedly creative, whether in the use of elaborate metaphors, in the creation of useful fictions, or in the formulation of thought experiments that try to test our best intuitions and explore the limits of conceivability and possibility. Let's look at the zombies argument (Chalmers 1996): zombies are perfect physical replicas of humans who pretend to do everything a human being does - they appear to feel anger, happiness, boredom or any mental state associated with human mental states. But, zombies do not really have anger, happiness or boredom, because they do not have any 
complex mental activity4. Now imagine a situation in which these creatures exist and think of the consequences: if you can conceive it, then this is possible5, and if it is possible, consciousness will have to be something other than physical states, for all the physical facts in this situation are the same as those physical facts that actually exist (given zombies are perfect physical replicas of humans). Nevertheless, zombies are not conscious. Chalmers's goal is to isolate a fundamental variable, consciousness, by conceiving creatures who possess physical properties but lack certain mental properties to argue against the reductionism of the mental to the physical, also known as reductionist physicalism.

It is clear that in scientific practice the notion of experiment is far distant and more rigorous than what is being discussed here, and it is hard to make sense of epistemological questions being descriptive or scientific in a Quinean fashion, but the aim of thought experiments do not differ much from the ultimate goal of isolating certain variables to build scenarios, in the sense we do not merely represent the phenomena we investigate passively, but create more or less correct informational models (semantic artefacts) of them, proactively and interactively (Floridi 2017).

Imagine an experiment to find out how a particular product for washing clothes clears textiles (Baggini e Fosl, 2010:85). In its everyday use there are a number of factors that can contribute to the product in question to behave in a certain way. Such factors include not only the active ingredients that are dissolved in the water, but also what types of fabrics will be cleansed, the temperature of the water, the parts of the washing machine, and so forth. Any experiment that aims to find out what caused the whitening of the textiles has to be planned in order to ensure that certain crucial factors are properly isolated from other variables. If, for example, we deal with the hypothesis that chlorine is the substance responsible for bleaching, the experiment needs to show that if all other factors are kept the same, then the presence or absence of chlorine will determine how the product whitens the fabrics. Thought experiments are based on the same principle, they try to isolate the variables that are crucial to the

${ }^{4}$ It is not plausible that they do not possess any brain activity whatsoever, since some of the basic skills such as locomotion and vision depend on brain activity.

${ }^{5}$ For a comprehensive article on epistemic and metaphysical possibilities see Anand (2016).

Manuscrito - Rev. Int. Fil. Campinas, v. 40, n. 4, pp. 77-96, out.dez. 2017. 
experiment, which, if present or absent, have a substantial specific effect on what is being analysed. The difference is that thought experiments do not test them, at least not at first, empirically, and their variables are isolated only through imagination.

In Putnam's (1975) Twin Earth example, relevant for the philosophy of language and philosophy of mind, only one constant is altered by changing the chemical compound that functions as water, "H2O" to "XYZ", and then considering the consequences of that change to the meaning of the word "water". Not only philosophers, but scientists also use thought experiments. It is well known that Einstein, for instance, used thought experiments in the elaboration of the Theory of Relativity. The difference is that in scientific enterprise generally the thought experiments lead to experimentation in laboratories. However, for philosophers most of times practical experiments are unnecessary, although there is the field of experimental philosophy which aims to combine traditional philosophical inquiry with systematic empirical inquiry. For most philosophers empirical experiments are unnecessary since the realm to be explored is usually the conceptual rather than the concrete. As the terrain to be explored here is also the conceptual, philosophical tools that explore and test our best intuitions are most welcome.

Back to the problem of logical omniscience, the failures of knowing or believing in all the consequences of what we actually know or believe seems to obviously derive from cognitive limitations. However, logical omniscience is a characteristic of an epistemic model which is conceived, in a minimally implicit way, as an idealization that possesses precisely the kind of motivations set forth above.

Another motivation to idealize is to simplify. The theoretical cost of describing and explaining every detail and particularity that we may observe in systems is very high. In most cases stick to complex features and variations is more harmful to research than abstracting them. The third motivation is normative. Even though our dynamics of states of belief and knowledge diverge from the ideal of logical omniscience, this is a state for which we tend to approach. This seems plausible, but there are well-established arguments in epistemology that demand that rationality requires recognition of our fallibility in relation to beliefs, roughly speaking, that rational agents must recognize that at least some of our beliefs or sets of beliefs are conflicting so that agents would be able to disbelief conjunctions of propositions. That being said, our fallibility does not necessarily contradicts the normative idea in which we "tend 
to an ideal state" or something near enough, but the issue at stake now is that to accept or deny normativity does not alter our scenario. Hence, it is not a very compelling reason to consider it as a main motivation or positive reason for idealization in standard epistemic logic.

The fourth and final motivation is pessimistic. Maybe the best we can do is to capture the logic of an idealized agent. We know how to account for a concept of knowledge that is applied to a model in which agents are deductively omniscient, but we cannot account for the many problems that we would have to face before a model that does not contain this consequence. I claim that the pessimistic view is a direct consequence of the scientific motivation, i.e., pessimism is an adverse consequence of our philosophical investigations rather than a motive per se. The ancient Skepticism revived after modernity, for example, was an important for questioning the possibility of knowledge and the limits of human nature from a cognitive point of view. Whether it is the Cartesian "evil demon" or the contemporary "brain in vat", skeptical challenges of such magnitude demonstrate the inverse spectrum of scientific motivation. If you cannot now be sure that you are not a brain in a vat, then you cannot rule out the possibility that all of your beliefs about the external world are false. Or, to put it in terms of knowledge claims: If you know that you are reading this text now, you know that you are not a brain in a vat, so (modus tollens) if you do not know that you are not a brain in a vat - for nothing in your experience can possibly reveal that you are such a brain, since your experience is identical ex hypothesi to that of someone or something that is not a brain in a vat -, so you do not know you're reading this text.

Both in the case of the omniscient agent or in the case of the agent deprived of any legitimate knowledge, such idealizations serve as arguments that are usually formulated through knowledge closure principle via the axiom $\mathrm{K}$ :

Given $\left(K_{a} p \wedge K_{a}(p \rightarrow q)\right) \rightarrow K_{a} q$

if a does not know that $q$

$\neg K_{a} q$

and a knows that pimplies $q$

$K_{a}(p \rightarrow q)$

Manuscrito - Rev. Int. Fil. Campinas, v. 40, n. 4, pp. 77-96, out.-der. 2017. 


\section{Then a does not know $p$ $\neg K_{a} p$}

Many epistemologists agree with the skeptic who resorts to the intuitive notion that the principle of closure of knowledge can be extended by deduction via modus tollens, but disagree with its results.

\section{Dynamic processes}

Fagin and Halpern (1988) pioneered an approach that distinguishes between implicit and explicit knowledge, and renders the agents immune from logical omniscience with respect to explicit knowledge. In this section I will sketch how that approach works differently within the notions of being and becoming informed, and then I present an example of a simplified model that fulfils the requirements of the four stated motivations and which contains the property of omniscience as the result of a dynamic process of an agent engaged in the action of transforming implicit into explicit knowledge.

On one hand we have standard normal modal logics (epistemic and doxastic) that works well with the technical notion of knowledge and belief (implicit), but which is inadequate to capture the ordinary notion of belief and knowledge. On the other hand, we have the ordinary and somewhat psychological notion of (explicit) knowledge and belief that tells us that we do not really believe in all the logical consequences of what we know, for we are affected by the following factors (Huang and Kwast 1991):

1. Awareness - As noted by Fagin and Halpern (1988), an agent can not claim to know or do not know that $p$ if $p$ is a concept about which he is not aware of. To put it in another way, an agent can not have explicit knowledge about what he is not aware of;

2. Limited resources - lack of computational resources and information processing prevent knowledge from becoming explicit; 
3. Irrelevance (or little importance) - an agent may not have a belief simply because of its irrelevance in a given context;

4. Lack of attention - self-explanatory, lack of attention or focus on important and relevant contexts, which can lead to not being in contact to contents and hence the agent not being capable of turning those contents explicit;

5. Prejudice - an agent may fail to make an explicit knowledge out of pure prejudice 6 .

The manifest fact that we are not logically omniscient comes from our cognitive limitations. We have idealized our agents too much, and the problem of logical omniscience does not seem to account for at least two salient points: First, in the semantics of epistemic logic the operator $\mathrm{K}$ seems to describe only the implicit semantic information of the agent, to which it definitely has the deductive closure property. The point that needs to be stressed here is that the closure property does not need to be preserved for a related notion, but intuitively different, that an agent is aware of some knowledge to be defined as explicit $[\operatorname{Ex} \varphi]$. When we ask ourselves how to define such a notion of explicit knowledge we are trying to account for the agents' attitudes.

The second point is to notice that the interesting problem is not knowing when explicit knowledge has deductive closure, but what agents have to do to turn implicit knowledge explicit. According to van Benthem and VelázquezQuesada (2010) when considering the premisses $\operatorname{Ex}(\varphi \rightarrow \psi)$ and $\operatorname{Ex} \varphi$, We say that the agent explicitly knows both $(\varphi \rightarrow \psi)$ and $\varphi$. As explicit knowledge must also be implicit knowledge, these should imply $\mathrm{K} \psi$, i.e., the agent knows implicitly $\psi$. To make such information explicit the agent must do some work, what van Benthem calls awareness raising, which leads to Ex $\psi$. When formalizing the distribution $\operatorname{Ex}(\varphi \rightarrow \psi) \rightarrow(\operatorname{Ex} \varphi \rightarrow \operatorname{Ex} \psi)$ it is inserted a gap [], so that we have:

$$
\operatorname{Ex}(\varphi \rightarrow \psi) \rightarrow(\operatorname{Ex} \varphi \rightarrow[] \operatorname{Ex} \psi)
$$

6 "But this confidence must be due to the speech itself, not to any preconceived idea of the speaker's character." Rhetoric (Aristotle 1991; I,2,1356a, 5-20). 
Where the gap represents the action to be added to turn knowledge explicit. To better understand the differences between the notions of explicit knowledge and implicit knowledge, semantic models are introduced, where propositional logic language is amplified by $\mathrm{K}$ operator, generating new formulas $\mathrm{K} \varphi$, which are read as "the agent knows that $\varphi$ " (Hintikka1962). As usual, we semantically consider that a set of possible worlds carries valuations with the accessibility relations indicating each possible world or situations for the agent. $\mathrm{K} \varphi$ is true in a world $\mathrm{w}$ if and only if it is true in all the worlds that the agent considers possible from w. Boolean connectives are also interpreted as usual. For the purpose here, a definition of the language to be used, a semantic model and at least one operation suffice. Formulas $\varphi$ of the language $L$ of epistemic awareness are given by:

$$
\text { Def. } \varphi: \because=\pi / A \varphi / \leftarrow \varphi / \varphi \wedge \psi / K \varphi
$$

$p \in \mathrm{P}$ (Set of atomic propositions). Other Boolean connectives $\vee, \rightarrow$ and $\leftrightarrow$ are defined in the usual way.

Formulas $A \varphi$ are read as "the agent is aware of $\varphi$ " and formulas $K \varphi$ are read as "the agent knows $\varphi$ implicitly.

The semantic model of epistemic awareness is defined as follows:

$$
\text { Def. } M=(W, R, A, V)
$$

Where $A: W \rightarrow \wp(\mathrm{L})$ is the awareness function that gives us the formulas that the agent "has in mind". According to Fagin and Halpern (1998) if $\varphi$ is known implicitly and considered explicitly we have:

$$
\operatorname{Ex} \varphi:=K(\varphi \wedge A \varphi)
$$

With regard to transitive and Euclidean accessibility, the following forms of introspection are obtained:

$$
\operatorname{Ex} \varphi \rightarrow K \operatorname{Ex} \varphi \quad(\text { positive) }
$$


$\neg \operatorname{Ex} \varphi \rightarrow K \neg \operatorname{Ex} \varphi$ (negative) ${ }^{7}$

And the operation that is important to us is that of consideration.

If $\mathrm{x}$ is any formula in $\mathrm{L}$ the model:

$M_{+x}=(W, R, A, V)$

is

$M=(W, \mathrm{R}, A, V)$ with the awareness sets extended by $\mathrm{x}$, which is:

$A^{\prime}(w):=A(w) \mathrm{U}\{x\}$ for each $w \in W$.

The results form an attempt to show, in addition to one of the many technical advances in the epistemic logic presented in the eighties and nineties by Fagin and Halpern and later by Huang and Kwast, van Benthem, among others, also the change in the approach in epistemology by the combination of formal and mainstream methods. Simple as it may seem to be, it was thanks to the recognition of the cognitive limitations of agents and the consequent deflation of their epistemic responsibilities that we could replace the excessively demanding requirements such as radical skepticism on the one hand, not treated in this paper, and extreme idealization on the other. A reticent objector might add that such approach may not be enough to exclude an evil demon, nor exonerate our agents from the excess of implicit information in our models. However, if knowledge has an inexorable commitment to truth, we can still add that infallible methods are not required to pursue knowledge.

${ }^{7}$ With the elimination of A6 and A8 axioms in IL such forms of introspection are not obtained (blind review ommitted). 


\section{Information excess in KTB-IL}

KTB-IL is not immune to the problem of information overload (blind review ommitted). The information overload is generated by including the inevitable rule of necessity along with the closure of the logic of being informed IL triggered by the inclusion of the axiom A58 (Floridi 2006), which in the informational version assumes the following form:

$$
I_{a}(\varphi \rightarrow \psi) \rightarrow\left(I_{a} \varphi \rightarrow I_{a} \psi\right)
$$

It also seems unrealistic to attribute to our information agent such a large amount of information, since the information agent a, thanks to the A5 + Nec is informed about all the provable theorems in the propositional calculus. Attempts to reconcile this undesirable result with KTB-IL system are also similar to the attempts described in the previous section, as it should be. If all normal modal logics have in their axiom schemata the axiom of distributivity and the rule of necessitation, then all normal modal logics share the problem of overload, be it epistemic, doxastic, deontic, temporal, or informational.

As argued earlier, to say that such logics deal with idealized agents (Lemmon 1959) is a rather ineffective manoeuvrer a la "biting the bullet", because it does not actually address the issue, it only prevents the problem from reaching its target. However, information logic (KTB-IL) has an advantage in comparison to other epistemic and doxastic systems. In an ideal (artificial) informational agent, e.g., a Turing Machine, what the rule of necessity is asserting is the conversion of $\varphi$. Instead of being a theorem, $\varphi$ can be inferred by an agent who, through the relevant axioms, can eventually deduce the information that $\varphi$ without an external input, at least in principle, a priori (Floridi, 2006:19).

Any sentence true in all scenarios (in the model) must be known by all agents in all of these scenarios. If what holds in a scenario is closed under logical consequence, then all logical consequences of what an agent knows must be known by that agent. As a particular instance of this phenomenon, all tautologies are known by all agents, regardless of the length of the tautology or the cognitive capacity (reasoning abilities, available memory, time in which to

${ }^{8} \mathrm{~A} 5$ in the Logic of Being Informed (Floridi, 2006), equivalent of $\mathrm{K}$.

Manuscrito - Rev. Int. Fil. Campinas, v. 40, n. 4, pp. 77-96, out.de\%. 2017. 
reason) of the agent. The suggestion I consider to immunize KTB-IL from omniscience regards the non-informative nature of logical truths, labelled as the Inverse Relationship Principle [IRP] (Barwise and Seligman 1997) and is directly related to the Bar-Hillel-Carnap semantic paradox [BCP] (1953):

It might perhaps, at first, seem strange that a self-contradictory sentence, hence one which no ideal receiver would accept, is regarded as carrying with it the most inclusive information. It should, however, be emphasized that semantic information is here not meant as implying truth. A false sentence which happens to say much is thereby highly informative in our sense. Whether the information it carries is true or false, scientifically valuable or not, and so forth, does not concern us. A self-contradictory sentence asserts too much; it is too informative to be true. (p. 229).

According to IRP, the probability $\mathrm{P}$ of $\varphi$, which has in its reach (image) sentences of any language, or events, situations, or worlds (Dretske 1981), is inversely proportional to the amount of semantic information carried by $\varphi$. Roughly, according to the classic theory of semantic information, there is more semantic content in "a circle is squared" than in the contingently true statement "France has a young president". Conversely, when $\varphi$ is a logical truth, we have that $\mathrm{P}(\varphi)=1$ and the degree of informativeness of $\varphi$ is 0 (zero), that is, $\operatorname{Inf}(\varphi)$ $=0$. Thus, when $\varphi$ is "empty", i.e., completely non-informative, as in the case of a tautology $(\varphi \vee \neg \varphi)$, as Wittgenstein pointed out in the Tractatus, then a holds $(\varphi \vee \neg \varphi)$, but can not be informed when receiving $(\varphi \vee \neg \varphi)$. The informational deficit of our agents can not be compensated by receiving a tautological message.

Information walks side by side with unpredictability. When the probability $\mathrm{P}$ for $\varphi$ is 1 , it is in the cases of tautology (always true), the degree of informativeness is zero. So with NEC we have:

\section{$\vdash \varphi \Rightarrow \vdash \mathrm{I} \varphi$}

The necessitation rule introduces a theorem $\vdash \varphi$, for a, that is in fact indistinguishable, since a already had the information that $\varphi$. We can interpret $\vdash \varphi \Rightarrow \vdash \mathrm{I} \varphi$ as an abbreviation of:

$$
\vdash \varphi \Rightarrow \mathrm{P}(\varphi)=1 \Rightarrow \operatorname{Inf}(\varphi)=0 \Rightarrow \vdash \mathrm{I} \varphi
$$


That does not mean that a is actually informed about all of the theorems from propositional calculus as well as from KTB-IL, as if he had a monstrous database through which he could consult to find the theorems, but it means that any provable theorem $\varphi$ in propositional calculus or in KTB-IL (true in all possible worlds) is not informative for a. A counter-intuitive consequence of the IRP is then illustrated by BCP. The less likely $\mathrm{p}$ is, the more informative $\mathrm{p}$ is, the more information it contains or can match. It seems that in the case of semantic information we have the truth encapsulated (Floridi 2004, blind review ommitted), i.e., instead of being possible to be true or not true (alethic condition), it is always true. Such view tells us that in logic a set of premises must contain all the information contained in the conclusion. "There can never be surprises in logic" (Wittgenstein 1994, \$6.1251). On the other hand, there is a strong intuition that to become informed an agent must be willing to act in a certain way, and this is only possible if an agent can, in the face of certain cognitive limitations, make information explicit from implicit information. The difference between the static notion of information and the dynamic notion being vs becoming informed - is nothing more than this disposition, this movement to make an information update from one state to another.

This result leads us back to the notion of "implicit knowledge" discussed in the previous section. We are not Turing Machines, nor do we have unlimited resources. It seems that the interesting problem in the case of information is no longer the causal closure for the provable theorems in the propositional calculus and KTB-IL. It is also no longer that of logical truths, since these, as we have seen, have a zero degree of informativeness. The interesting problem now is to know what agents have to do to turn implicit information in explicit information. Our model will turn out to be similar to that of knowledge, since in most cases both notions collapse when not implicit:

$$
\mathrm{M}=(W, \mathrm{R}, A, V)
$$

Where $A: W \rightarrow \wp(L)$ is the awareness function that gives us the formulas that the agent "has in mind", but could also be in a hard drive, with the regard that in explicit cases it was endorsed by the agent (blind review ommitted).

If an agent is informed that $\varphi$ implicitly (that information is available on the network) and $\varphi$ is considered explicitly we have (the agent accessed the information):

Manuscrito - Rev. Int. Fil. Campinas, v. 40, n. 4, pp. 77-96, out.-der. 2017. 
$\operatorname{Ex} \varphi:=I(\varphi \wedge A \varphi)$

Being informed that $\varphi$ and becoming informed that $\varphi$ are different notions. The first is static, we have the informational state of an agent at a particular time. One way of dealing with information through logical analysis is to devise the informational (static) state of an agent as the set of all relevant possibilities that the agent hold. If an agent a is informed that $(p \vee q)$, but does not know which of the disjoints is true, Then there are three ways that the world could be, from all the information that a holds. Assuming that negation behaves in a classical way, we can talk about three possible worlds: $p\urcorner p, \neg p q$ and $p q$. The informational state of the agent $\mathrm{a}$ is that he can not discriminate between certain possible worlds, for he can not say for certain which of the worlds is the actual one, a case of "epistemic indistinguishability" for the agent (Stalnaker, 2008). This relation of indistinguishability can be illustrated as a relation $\sim$ in as many possible worlds, so that at least $w 1 \sim a w 2$ means that the information held by a it is not sufficient to distinguish between $w 1$ and $w 2$.

\section{Final Considerations}

The larger the class of indistinguishable worlds, the less information the agent possesses. Information, in this regard, can be understood as a tool that an agent can use to discriminate the current world from the merely possible ones. Therefore, the dynamic sense of information is a kind of bottleneck or filtering in the class of indistinguishable worlds. Genuine (true) information never excludes the current world in this filtering process, but misinformation can cause an agent to consider certain things over others that should in fact be considered. The information contained in declarative statements is the cause of a change in the information state of an agent. Therefore, we must model the information content of $p$ as an update of the indistinguishability relation, so that after updating, agent a can distinguish which worlds in which $p$ holds from those in which $p$ is no longer the case.

What can be misleading is that the kind of world we are talking about can not be that of the traditional philosophical notion of the metaphysically possible world. Possible worlds framework allows us to introduce a set of modal notions: a proposition is necessary only in case it is true in all possible 
worlds, a proposition is possible only in case it is true in some possible worlds, and it is contingent only in case it is true in some but not all possible worlds. A sentence is necessary only in case it expresses a necessary proposition. But for the relevant information updates discussed, the sense of possibility must be epistemic as in the notions we employ when we say things like "the train to London can be delayed" or "Julian Assange must be tired". These modal utterances seem to make claims about what the available evidence shows, or about which scenarios can be ruled out on the basis of the evidence. Then, we can only make sense of unrestricted cognitive capacities a la ideal agents in cases like: $p$ is epistemically necessary for an agent a just in case the empirical evidence a possesses and ideal reasoning rules out $\neg p$.

It is way beyond the reach of this paper to get into the controversial question whether the necessary propositions are all and only the epistemically necessary (a priori) ones, or whether the extensions of the two concepts can come apart (Kripke 1980, Putnam 1975), but certainly it is interesting to combine modal notions with an account of semantic content. It is also important to notice that epistemic possibilities are (in part) psychological notions. Beliefs and desires in fact exist and according to Dennet psychological notions "(...) can be discerned only from the point of view of one who adopts a certain predictive strategy, and [their] existence can be confirmed only by an assessment of the success of that strategy." (Dennett, 1987:15).

It seems that information updating is part of a prediction strategy and it is clear that humans have expanded the predictive capabilities thanks to the technological advances, dissemination of information and communication. However, we have also seen that the extreme idealization of agents does not seem to be adequate and that the representation of knowledge involving quantifiers escaped for many years a satisfactory immunization against logical omniscience.

\section{References}

Allo, P. "The Logic of 'Being Informed' revisited and revised", Philosophical Studies, 2011.

ANAND, V. "The Epistemology of Modality", The Stanford Encyclopedia of Philosophy (Winter Edition), Edward N. Zalta (ed.), URL = 
$<\underline{\text { https://plato.stanford.edu/archives/win2016/entries/modality- }}$ epistemology/>, 2016.

ARISTOTLE, On Rhetoric, newly translated by George A. Kennedy, Oxford University Press, New York, 1991.

BAggini, L. e FosL, P.S. The Philosophers Toolkit - A Compendium of Philosophical Concepts and Methods. Wiley-Blackwell, USA, 2010.

BAR-Hillel, Y. e CARnAP, R. "An Outline of a Theory of Semantic Information", repr. in Bar-Hillel [1964], 221-74, 1953.

BARWISE, J. and SEligman, J. Information Flow: The Logic of Distributed Systems, Cambridge: Cambridge University Press, 1997.

Chalmers, D. J. The Conscious Mind: In Search of a Fundamental Theory. Nova Iorque, Oxford University Press, 1996.

Chomsky, NoAm. Aspects of the Theory of Syntax. Cambridge, MA: MIT Press, 1965.

DENNETT, D. The Intentional Stance. MIT Press, 1985.

“Can Machines Think?” in: Brainchildren: Essays on Designing Minds, 1998.

MIT Press, Cambridge MA. 3-20.

FAGIN R., Halpern J. Y, Moses Y. e Vardi M. Y. Reasoning about knowledge, MIT Press, Cambridge, Mass, 1995.

FLORIDI, L. "Outline of a Theory of Strongly Semantic Information". Minds and Machines 14: 197, Springer, 2004.

"The Logic of Being Informed". Logique et Analyse, 49, pp. 433-460, 2006.

"Semantic Information and the Correctness Theory of Truth", Erkenntnis, 74 (2), 147-175, 2010. "A Plea for Non-naturalism as Constructionism". Minds and Machines, 27(2), 269-285, Springer, 2017.

Hendricks, V. F. Mainstream and Formal Epistemology, Cambridge University Press. 
HintikKA, J. (1962), Knowledge and Belief, Cornell University Press, 2006.

"A second generation epistemic logic and its general significance", in V.

F. Hendricks, S. A. Pedersen, and K. F. Jørgensen (eds.), Knowledge Contributors, number 322 in Synthese Library. Kluwer Academic, Dordrecht, 2003.

HUANG, Z.S. and K. KWAST. "Awareness, negation and logical omniscience", in J. van Eijck (Ed.), Logics in AI, volume 478 of Lectures Notes in Computer Science, pages 282-300, Springer, 1991.

Lemmon, E. J. "Review: K. Jaakko, J. Hintikka, Quantifiers in Deontic Logic." J. Symbolic Logic 24, no. 2, 178-179, 1959.

LeVesque, H. J. "A logic of implicit and explicit belief", in: Proceedings AAAI84 Austin, TX, 198-202, 1984.

JAGO, M. "Hintikka and Creswell on Logical Omniscience", Logic and Logical Philosophy, 15(1), 325-354, 2006.

KRIPKE, S. Naming and Necessity. Oxford, Blackwell, 1980.

Putnam, H. "The Meaning of 'Meaning". Language, Mind and Knowledge, Minnesota Studies in the Philosophy of Science, VII, Minneapolis: University of Minnesota Press, 1975.

Quine, W. V. Theories and Things. Cambridge, Mass.; London: Belknap Press of Harvard University Press, 1981.

SEARLE, J. R. "Is the Brain a Digital Computer?" Proceedings and Addresses of the American Philosophical Association, 64, 21-37, 1990.

StAlnaker, R. "The Problem of Logical Omniscience", in: Synthese, 89(3), Belief and Rationality, 425-440, Springer, 1991.

Our Knowledge of the Internal World, Nova Iorque, Oxford University Press, 2008.

VAN Benthem, J. Logical dynamics of information and interaction. Cambridge, Cambridge University Press, 2010.

VelázQuez-QuesadA, F. R. e VAN BENTHEM, J. "The dynamics of awareness". Synthese, 177 (1), 5-27, 2010.

Manuscrito - Rev. Int. Fil. Campinas, v. 40, n. 4, pp. 77-96, out.de\%. 2017. 
WitTGenstein, L. Tractatus Logico-Philosophicus. Trad., notas e ensaio introdutório de Luiz Henrique L. Santos. 2. ed. São Paulo: Edusp, 1994. 